\title{
Expression of the erythropoietin receptor in patients with proliferative diabetic retinopathy and its correlation with postoperative visual prognosis
}

\author{
Guiqin $\mathrm{He}^{1}$, Junhong Zhou ${ }^{2}$, Xiaoli Yang ${ }^{1}$ \\ ${ }^{1}$ Department of Ophthalmology, Affiliated Hospital of North Sichuan Medical College, Nanchong, China; ${ }^{2}$ Department of Orthopedics, Affiliated \\ Hospital of North Sichuan Medical College, Nanchong, China \\ Contributions: (I) Conception and design: G He, X Yang; (II) Administrative support: J Zhou; (III) Provision of study materials and patients: G He, J \\ Zhou; (IV) Collection and assembly of data: All authors; (V) Data analysis and interpretation: G He, X Yang; (VI) Manuscript writing: All authors; (VII) \\ Final approval of manuscript: All authors. \\ Correspondence to: Xiaoli Yang. Department of Ophthalmology, Affiliated Hospital of North Sichuan Medical College, Nanchong 637000, China. \\ Email: heguiqin18@163.com.
}

Background: Proliferative diabetic retinopathy (PDR) is a leading cause of blindness. This study aimed to analyze the expression of the erythropoietin receptor (EpoR) in patients with PDR and its correlation with postoperative visual prognosis.

Methods: Between May 2016 and May 2017, 89 patients who underwent vitreoretinal surgery (VRS) in Affiliated Hospital of North Sichuan Medical College were enrolled. Among them, 45 patients (52 eyes) with PDR and 44 patients (50 eyes) with retinal vein occlusion (RVO) made up the study group and the control group, respectively. All patients received $25 \mathrm{G}$ standard flattened three-channel lens-preserving VRS; all procedures were performed by the same physician. Recovery and change in best corrected visual acuity (BCVA) were observed. Subsequently, the patients were divided into group A (improved vision) and group B (no improvement in vision). The integrated optical density (IOD) value of EpoR expression among the groups were compared. Pearson's correlation analysis was used to analyze the correlation between the IOD values of EpoR and the change in visual acuity after surgery for PDR.

Results: The IOD value of EpoR in the study group was higher than that in the control group $(\mathrm{P}<0.05)$. The postoperative BCVA of the PDR patients was significantly higher than that before surgery $(\geq 0.1 v s$. $\leq 0.02, \mathrm{P}<0.05$ ). Of the $45 \mathrm{PDR}$ patients (52 eyes), 34 patients (39 eyes) had improved visual acuity after surgery (group A), while 11 patients (13 eyes) experienced no improvement (group B). The IOD value of EpoR expression in group $\mathrm{B}$ was higher than that in group $\mathrm{A}(\mathrm{P}<0.05)$. Pearson's correlation analysis showed that the IOD value of EpoR expression were positively correlated with the recovery.

Conclusions: EpoR is expressed at a high level in PDR patients. The expression level of EpoR in the epiretinal membrane of PDR patients is positively correlated with the prognosis of postoperative vision. Therefore, EpoR has promise as a prognostic biomarker and a potential therapeutic target for PDR.

Keywords: Proliferative diabetic retinopathy (PDR); EpoR; postoperative visual prognosis; correlation analysis

Submitted Sep 03, 2020. Accepted for publication Nov 04, 2020.

doi: 10.21037/apm-20-1898

View this article at: http://dx.doi.org/10.21037/apm-20-1898 


\section{Introduction}

Retinopathy, or diabetic retinopathy (DR), is the most common microvascular complication in patients with diabetes. DR results from ischemic and hypoxic changes in the eye caused by the prolonged elevation of blood sugar levels (1), and is usually divided into two types: non-proliferative and proliferative. Proliferative diabetic retinopathy (PDR) manifests as the formation of new blood vessels, fibrous proliferation, and even retinal detachment (2). Retinal neovascularization plays a pivotal role in the development of PDR and has long been the focus of clinical attention.

Erythropoietin (Epo) is a hormone-like substance that can promote the production of red blood cells in the body under bone marrow hematopoiesis (3). The results of in vitro experiments have shown that Epo can promote angiogenesis by binding with its receptor, erythropoietin receptor (EpoR), to stimulate the proliferation and migration of endothelial cells and promote angiogenesis; consequently, its role in the occurrence and development of PDR has attracted much attention (4). This study aimed to analyze the expression of EpoR in patients with PDR and its correlation with postoperative visual prognosis in order to provide novel ideas for the clinical treatment of PDR. We present the following article in accordance with the STROBE reporting checklist (available at http://dx.doi. org/10.21037/apm-20-1898).

\section{Methods}

\section{General information}

Eighty-nine patients with epiretinal membrane who underwent vitreoretinal surgery (VRS) in our hospital between May 2016 and May 2017 were enrolled. The study participants included 45 patients with PDR (52 eyes) and 44 patients (50 eyes) with retinal vein occlusion (RVO), who made up the study group and the control group, respectively. The study group comprised 25 males (30 eyes) and 20 females (22 eyes) with an average age of $51.32 \pm$ 3.91 years (range, 32-72 years). The control group comprised 24 males (27 eyes) and 20 females (23 eyes) with an average age of $51.71 \pm 3.67$ years (range, $30-73$ years). No significant differences existed in the general information between the two groups $(\mathrm{P}>0.05$, Table 1$)$.

The inclusion criteria were: (I) preretinal fibrovascular membrane proliferation accompanied by traction retinal detachment and/or vitreous hemorrhage; (II) in patients with invisible fundus before surgery, confirmation of the existence of the preretinal proliferative membrane through observation of the fundus under an operating microscope after the surgical removal of the vitreous ; (III) good control of the general condition; and (IV) agreed to participate in this study and signed the informed consent form. This study was approved by the medical ethics of the committee of Affiliated Hospital of North Sichuan Medical College [No. 2019ER(R)107-1]. The exclusion criteria were as follows: (I) a past history of eye surgery or laser treatment; (II) a history of blood diseases, rheumatic diseases, immune system diseases, or other severe disorders that could affect the normal plasma secretion of Epo; (III) patients with dementia or mental health disorders who were unable cooperate with the study. All procedures performed in this study involving human participants were in accordance with the Declaration of Helsinki (as revised in 2013).

\section{Surgical methods}

All patients received $25 \mathrm{G}$ standard flattened three-channel lens-preserving VRS; all procedures were performed by the same physician. During the operation, the vitreous body and the retinal surface proliferative membrane were removed entirely. The tightly adhered fibrous vascular membrane was released, and an incision were made on the hard retinal folds as appropriate. Bleeding in patients with repeated hemorrhage due to neovascularization was stopped with intraocular electrocoagulation. The retina was reattached with heavy water or air/liquid exchange, and intraocular retinal laser photocoagulation or laser photocoagulation was performed after surgery. Finally, the filler was injected according to the position of the retinal hole and the change in the fundus. Patients were followed up for more than 3 months postoperatively, and their recovery and changes in best corrected visual acuity (BCVA) were observed.

\section{Main equipment and reagents}

The microscope was purchased from Olympus (Japan). An immunohistochemistry streptavidin peroxidase (SP) staining kit, a 3,3'-Diaminobenzidine (DAB) chromogenic kit, phosphate-buffered saline (PBS, dry powder), bovine serum albumin, biotinylated conjugated goat anti-rabbit immunoglobulin G (IgG), citric acid, and sodium citrate (dry powder) were obtained from Zhongshan Jinqiao Bio Technology (Beijing, China). Rabbit anti-human EodR primary antibody was purchased from Beijing Boaosen 
Table 1 Comparison of the integrated optical density values of erythropoietin receptor expression between the study group and the control group

\begin{tabular}{lcccc}
\hline Item & Study group & Control group & $t$ & $\mathrm{P}$ \\
\hline $\mathrm{N}$ (eyes) & 52 & 50 & & \\
IOD value & $0.0179 \pm 0.004$ & $0.0113 \pm 0.003$ & 9.399 & $<0.001$ \\
\hline
\end{tabular}

Biotechnology Ltd. (Beijing, China).

\section{Detection of EpoR expression}

During the operation, the stripped epiretinal membrane was collected, and promptly placed in $4 \%$ formaldehyde solution for fixing for 1-2 days. Following dehydration with ethanol at different concentrations, the tissues were placed in xylene, and then immersed in paraffin. The specimens were cut into continuous $4-\mu \mathrm{m}$-thick slices, heated in an oven at $70^{\circ} \mathrm{C}$ for 1 hour, and then subjected to conventional hematoxylin and eosin staining. The morphology of the epiretinal membrane was observed under an optical microscope. After deparaffinization, dehydration, and rehydration, the tissue sections were heated in citrate buffer for antigen repair, and a 3\% ( $\mathrm{v} / \mathrm{v})$ hydrogen peroxide solution was used to block endogenous peroxidase activity. Next, the sections were blocked with goat serum at room temperature for 30 minutes. The solution was discarded (without washing), and the sections were incubated with primary antibody for EpoR (mouse anti-human antibody) at $4{ }^{\circ} \mathrm{C}$ overnight. Then the sections were incubated at $37^{\circ} \mathrm{C}$ for 1.5 hours, the sections were washed with PBS before human biotin-conjugated goat anti-mouse IgG antibody was added and the sections were incubated at $37^{\circ} \mathrm{C}$ for 30 minutes. The sections were washed again with PBS and incubated with horseradish peroxidase (HRP)conjugated streptavidin working solution at $37{ }^{\circ} \mathrm{C}$ for 30 minutes. Then, the sections were washed with PBS, treated with $\mathrm{DAB}$ for 30 minutes for color development, and counterstained with hematoxylin for 30 seconds. Following this, the tissues were subjected to gradient ethanol dehydration, xylene transparency and neutral gum mounting. Tissues treated with PBS rather than primary antibody were used as a blank control.

For each slice of epiretinal membrane with complete structure, five high power fields $(\times 400)$ were randomly selected, and images were obtained using the microscope. Image Pro Plus 6.0 software (Media Cybernetics, Maryland,
USA) was used to analyze the average cumulative optical density value with the integrated optical densities (IOD) for each slice, and the expression of EpoR in the epiretinal membrane of each slice was quantitatively analyzed.

\section{Measurement of BCVA (5)}

The BCVA of all patients was assessed before and 28 days after surgery using the standard logarithmic visual acuity chart. Visual acuity was assessed with the patient positioned within 2 meters of the chart, and the number of BCVA letters read was obtained according to the standard optometry procedure. The data were recorded and analyzed.

\section{Statistical analysis}

Data were statistically analyzed using SPSS22.0 software (IBM, New York, USA). Measurement data were expressed as mean \pm standard deviation, and analyzed using $t$ tests or $\chi^{2}$ tests. Pearson's correlation analysis was used to assess the correlation between the IOD values of EpoR and the change in visual acuity after surgery for PDR. $\mathrm{P}<0.05$ was considered to show statistical significance.

\section{Results}

Comparison of IOD values of EpoR expression between the study group and the control group

The IOD value of EpoR expression in the study group was significantly higher than that in the control group $(\mathrm{P}<0.05$, Table 1).

\section{Comparison of BCVA before and after PDR}

The postoperative BCVA of the PDR patients was significantly higher than that before surgery $(\geq 0.1 v s . \leq 0.02$, $\mathrm{P}<0.05)$. The immunohistochemical detection images in two groups are shown in Figure 1. The postoperative BCVA of PDR patients was significantly higher following surgery $(\mathrm{P}<0.05$, Figure 1, Table 2).

\section{Comparison of the IOD value of EpoR expression in group $A$ and group $B$}

Of the 45 patients with PDR, 34 patients (39 eyes) showed an improvement in visual acuity after surgery, while the 

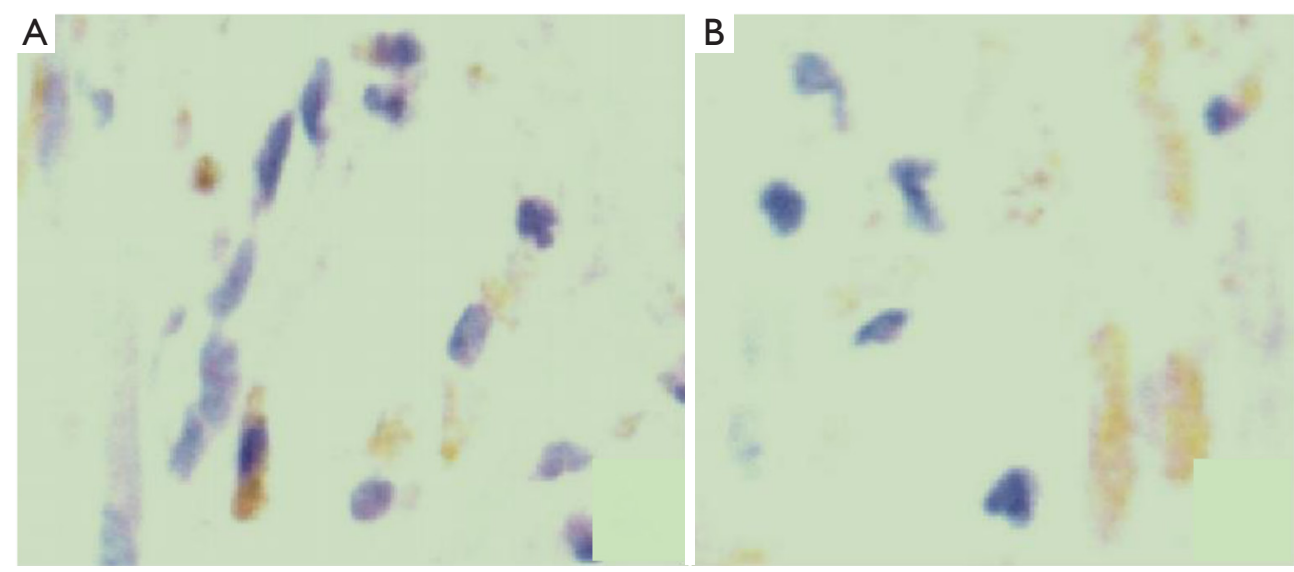

Figure 1 EpoR expression in the anterior retinal membrane of the two groups (immunohistochemical staining, $\times 400$ ). (A) The expression of erythropoietin receptor (EpoR) expression in the anterior retinal membrane of patients with proliferative diabetic retinopathy; (B) expression of EpoR in the anterior retinal membrane of patients with retinal vein occlusion.

Table 2 Comparison of best corrected visual acuity (BCVA) between patients with proliferative diabetic retinopathy before and after surgery

\begin{tabular}{|c|c|c|c|c|c|}
\hline Item & No. of eyes & \multicolumn{3}{|c|}{ BCVA distribution, $\mathrm{n}(\%)$} & Average BCVA \\
\hline Before surgery & 52 & $8(15.38)$ & $15(28.85)$ & $29(55.77)$ & $0.31 \pm 0.05$ \\
\hline After surgery & 52 & $32(61.54)$ & $14(26.92)$ & $6(11.54)$ & $0.57 \pm 0.07$ \\
\hline$t / \chi^{2}$ & & 23.400 & 0.048 & 22.781 & 217.951 \\
\hline
\end{tabular}

Table 3 Comparison of the integrated optical density (IOD) values of erythropoietin receptor expression in group A and group B $\left(\bar{x}_{ \pm} \mathrm{S}\right)$

\begin{tabular}{lcccc}
\hline Item & Group A & Group B & $t$ & $P$ \\
\hline N (eyes) & 39 & 13 & & \\
IOD value & $0.0140 \pm 0.005$ & $0.0189 \pm 0.007$ & 4.080 & $<0.001$ \\
\hline
\end{tabular}

remaining 11 patients (13 eyes) showed no improvement. According to whether their visual acuity had improved or not, the patients were classified into group A and group B, respectively. The IOD value of EpoR expression in group $\mathrm{B}$ was higher than that in group $\mathrm{A}(\mathrm{P}<0.05$, Table 3).

\section{Correlation analysis of IOD value of EpoR expression and visual acuity changes after PDR}

The correlation between BCVA and the IOD value of EpoR expression in the PDR patients after surgery was analyzed. The results revealed that the two were positively correlated $(\mathrm{r}=0.533, \mathrm{P}<0.05$, Figure 2$)$.

\section{Discussion}

PDR is a leading cause of blindness that has been a research hotspot in recent years. PDR has a complex pathogenesis that involves many risk factors, including growth factors, genetic predispositions, and autoimmune reactions (6). At present, it is believed that the pathogenesis of PDR includes many aspects such as high osmotic pressure, hyperglycemia, vascular growth factor. High osmotic pressure could cause relatively low intraocular pressure and changes in the morphology and function of retinal pigment epithelial cells, which is the key to the pathogenesis of PDR. And the hyperglycemia in diabetic patients can lead to relatively low intraocular pressure. When the intraocular pressure is reduced, the blood vessel perfusion pressure can be relatively increased, promoting the dilation of small veins and the increase of retinal blood flow, further damaging 


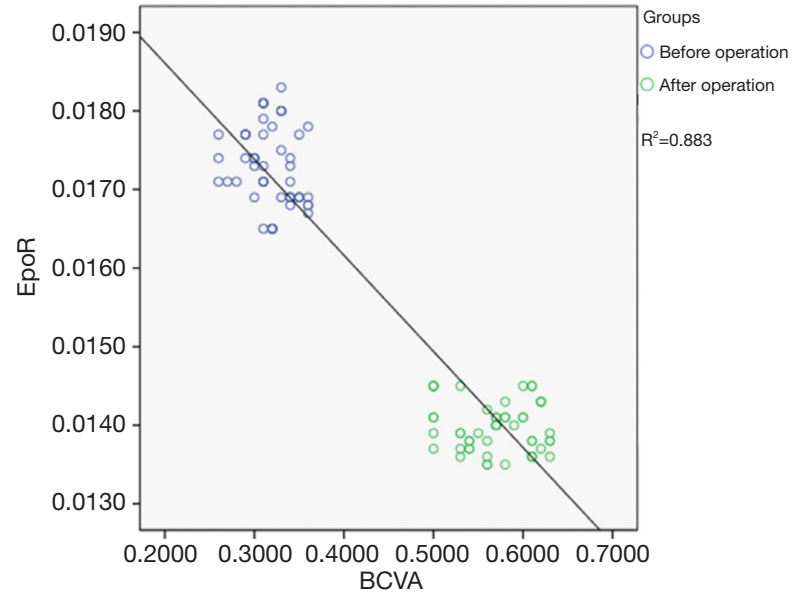

Figure 2 Correlation analysis between the integrated optical density integrated optical density (IOD) value of erythropoietin receptor erythropoietin receptor (EpoR) expression and changes in visual acuity after surgery in patients with proliferative diabetic retinopathy. BCVA, best corrected visual acuity.

the self-regulation effect of diabetic patients. At the same time, the increase of patient's intraocular perfusion pressure may increase the extravasation of the retinal lesion vessels, and then manifest as the exudative changes of DR. Longterm effusion changes can increase the pressure on the blood vessel wall, making the blood vessel wall thinner and prone to proliferative lesions (7). In addition, under normal circumstances, vascular proliferation factor and vascular proliferation inhibitor are in a dynamic balance, whether the vascular proliferation factor is activated or increased, or the inhibitory factor is inhibited or reduced, will break the original balance, resulting in angiogenesis. Both PDR and RVO belong to hypoxic retinopathy. In patients with these two diseases, large-area ischemia of the retina can cause neovascularization of the retina and the optic disc, the iris, and the anterior chamber angle, which can lead to numerous disorders such as retinal hemorrhage, vitreous hemorrhage, proliferative vitreoretinopathy, traction retinal detachment, and neovascularization of the eyes (8). The onset of neovascular retinopathy sees the active proliferation of new blood vessels; these fragile new vessels cause vitreous hemorrhage or fluid leakage, resulting in a further decrease in vision (9). As the disease progresses, the proliferating blood vessels begin to become fibrotic and curled, leading to complications such as retinal detachment and, eventually, vision loss (10).

A number of recent studies have found that a range of growth factors contribute to the occurrence of neovascularization. Therefore, it is of great significance to discover a target for the prevention and treatment of vision damage caused by PDR, based on its molecular biological mechanisms (11). Epo is a glycoprotein hormone that can stimulate the hematopoietic system. Specifically, after binding to EpoR on the surface of erythroid progenitor cells and proto-erythrocytes, Epo can promote cell proliferation and differentiation to form functionally mature red blood cells. Furthermore, Epo promotes capillary formation without weakening the tight junctions between endothelial cells and thus does not increase exudation $(12,13)$. Therefore, Epo and its receptor, EpoR, have gradually attracted attention as independent factors promoting retinal neovascularization (14). Here, in order to study the role of EpoR in PDR, this study analyzed the IOD values of EpoR expression in the vitreous of 45 PDR patients (52 eyes) and 44 RVO patients (50 eyes). The IOD value of EpoR expression was found to be higher in the study group than in the control group $(\mathrm{P}<0.05)$, suggesting that the EpoEpoR pathway plays an important role in the formation of the epiretinal membrane in PDR.

Further, this study also explored the correlation between the expression level of EpoR in the epiretinal membrane of PDR patients and postoperative visual prognosis. The results showed that PDR patients who had no improvement in visual acuity postoperatively had a higher expression level of EpoR in the epiretinal membrane than those patients with improved visual acuity. Pearson's correlation analysis also showed that EpoR expression and postoperative visual acuity were positively correlated with each other $(\mathrm{P}<0.05)$, which may be related to the obvious proliferation ability of the epiretinal membrane in PDR patients. The results of this study also suggest that clinically, it is possible to inhibit the angiogenesis-promoting effect of Epo by blocking the binding of Epo and EpoR, thereby inhibiting PDR neovascularization and proliferation of the epiretinal membrane $(15,16)$. In addition, relevant studies have shown that risk factors for postoperative vision in PDR patients also include vitreous hemorrhage, retinal detachment and secondary glaucoma. Therefore, the clinical operation time should be strictly controlled, and the surgical treatment should be carried out in time when the macula is not obviously involved, so as to ensure the postoperative vision recovery to a more satisfactory degree (17). Some studies have also suggested that poor vision after PDR is related to multiple factors including insulin use, patient compliance, preoperative application of anti-VEGF drugs, preoperative 
treatment points and other factors.

In conclusion, EpoR is highly expressed in PDR patients. The expression level of EpoR in the epiretinal membrane of PDR patients is positively correlated with postoperative visual prognosis, which suggests the potential of EpoR as a prognostic biomarker for PDR patients as well as a target for PDR treatment. However, the limitation of this study is that the sample size is small, and this study selects only a set of RVO patients as control, but without collecting other retinal membrane such as idiopathic retinal membrane before, idiopathic macular hole and PVR retinal membrane as normal control group. The main difficulties are that the retinal membrane mentioned above is thin, fixed difficulty, and easy to be lost in the immunohistochemical process of middling dewaxing, which may have larger influence on the accuracy of the results. Another shortcoming is that this study only chose EpoR as test object, not to collect vitreous fluids and detect the Epo levels, and Epo expression of membrane in front of the retina were also not measured, which might be of poor persuasion. But in the past, a lot of studies have reported the level of Epo in vitreous cavity. And the other reason is due to the limitation of funding, we only detect the expression of EpoR.

\section{Acknowledgments}

Funding: None.

\section{Footnote}

Reporting Checklist: The authors have completed the STROBE reporting checklist. Available at http://dx.doi. org/10.21037/apm-20-1898

Data Sharing Statement: Available at http://dx.doi. org/10.21037/apm-20-1898

Conflicts of Interest: All authors have completed the ICMJE uniform disclosure form (available at http://dx.doi. org/10.21037/apm-20-1898). The authors have no conflicts of interest to declare.

Ethical Statement: The authors are accountable for all aspects of the work in ensuring that questions related to the accuracy or integrity of any part of the work are appropriately investigated and resolved. This study was approved by the Affiliated Hospital of North Sichuan Medical College [No. 2019ER(R)107-1], and informed consent was taken from all the patients. All procedures performed in this study involving human participants were in accordance with the Declaration of Helsinki (as revised in 2013).

Open Access Statement: This is an Open Access article distributed in accordance with the Creative Commons Attribution-NonCommercial-NoDerivs 4.0 International License (CC BY-NC-ND 4.0), which permits the noncommercial replication and distribution of the article with the strict proviso that no changes or edits are made and the original work is properly cited (including links to both the formal publication through the relevant DOI and the license). See: https://creativecommons.org/licenses/by-nc-nd/4.0/.

\section{References}

1. Ranjan A, Chowdhary CL, Gurani VK. Diabetic Retinopathy Detection Using Neural Network - August 2019. International Journal of Innovative Technology and Exploring Engineering 2019;71:1457-60.

2. Ljubimov AV, Burgeson RE, Butkowski RJ, et al. Basement membrane abnormalities in human eyes with diabetic retinopathy. J Histochem Cytochem 1996;44:1469-79.

3. Miljus N, Massih B, Weis MA, et al. Neuroprotection and endocytosis: erythropoietin receptors in insect nervous systems. J Neurochem 2017;141:63-74.

4. Nesper PL, Roberts PK, Onishi AC, et al. Quantifying Microvascular Abnormalities With Increasing Severity of Diabetic Retinopathy Using Optical Coherence Tomography Angiography. Invest Ophthalmol Vis Sci 2017;58:BIO307-15.

5. Maiese K. Warming Up to New Possibilities with the Capsaicin Receptor TRPV1: mTOR, AMPK, and Erythropoietin. Curr Neurovasc Res 2017;14:184-9.

6. Chan KK, Matchett KB, Coulter JA, et al. Erythropoietin drives breast cancer progression by activation of its receptor EPOR. Oncotarget 2017;8:38251-63.

7. Rübsam A, Parikh S, Fort PE. Role of Inflammation in Diabetic Retinopathy. Int J Mol Sci 2018;19:942-6.

8. Landau D, London L, Bandach I, et al. The hypoxia inducible factor/erythropoietin (EPO)/EPO receptor pathway is disturbed in a rat model of chronic kidney disease related anemia. PLoS One 2018;13:e0196684.

9. Foley LS, Fullerton DA, Mares J, et al. Erythropoietin's Beta Common Receptor Mediates Neuroprotection in Spinal Cord Neurons. Ann Thorac Surg 2017;104:1909-14.

10. Hansjürgen A, Martin G, Ziemssen A. Pathomechanismen 
der diabetischen Retinopathie; Pathomechanisms of diabetic retinopathy. Der Diabetologe 2018;14:542-9.

11. Fecková B, Kimáková P, Ilkovičová L, et al. Methylation of the first exon in the erythropoietin receptor gene does not correlate with its mRNA and protein level in cancer cells. BMC Genet 2019;20:1.

12. Castillo J, Aleman I, Rush SW, et al. Preoperative Bevacizumab Administration in Proliferative Diabetic Retinopathy Patients Undergoing Vitrectomy: A Randomized and Controlled Trial Comparing Interval Variation. Am J Ophthalmol 2017;183:1-10.

13. Li C, Lanman NA, Kong Y, et al. Inhibition of the erythropoietin-producing receptor EPHB4 antagonizes androgen receptor overexpression and reduces enzalutamide resistance. J Biol Chem 2020;295:5470-83.

14. Jain A, Prasad P, Chaudhry S, et al. A novel mutation in erythropoietin receptor gene (c.1308_1309insG) in an Indian patient with erythrocytosis. Eur J Haematol

Cite this article as: $\mathrm{He}$ G, Zhou J, Yang X. Expression of the erythropoietin receptor in patients with proliferative diabetic retinopathy and its correlation with postoperative visual prognosis. Ann Palliat Med 2020;9(6):4010-4016. doi: 10.21037/ apm-20-1898
2019;103:449-50.

15. Durbin MK, An L, Shemonski ND, et al. Quantification of Retinal Microvascular Density in Optical Coherence Tomographic Angiography Images in Diabetic Retinopathy. JAMA Ophthalmol 2017;135:370-6.

16. Hernández CC, Burgos CF, Gajardo AH, et al. Neuroprotective effects of erythropoietin on neurodegenerative and ischemic brain diseases: the role of erythropoietin receptor. Neural Regen Res 2017;12:1381-9.

17. Guo J, Chen S, Wang Y, et al. Clinical effect of vitreoretinal surgery combined with intravitreal injection of conbercept or ranibizumab on severe proliferative diabetic retinopathy. Zhonghua Shiyan Yanke Zazhi/ Chinese Journal of Experimental Ophthalmology 2017;35:914-9.

(English Language Editor: J. Reynolds) 